\title{
Variations in the work function of doped single- and few-layer graphene assessed by Kelvin probe force microscopy and density functional theory
}

\author{
D. Ziegler, ${ }^{1, *}$ P. Gava, ${ }^{2}$ J. Güttinger, ${ }^{3}$ F. Molitor, ${ }^{3}$ L. Wirtz,${ }^{4}$ M. Lazzeri, ${ }^{2}$ A. M. Saitta, ${ }^{2}$ A. Stemmer, ${ }^{1}$ \\ F. Mauri, ${ }^{2}$ and C. Stampfer ${ }^{3,5}$ \\ ${ }^{1}$ Nanotechnology Group, ETH Zurich, 8092 Zurich, Switzerland \\ ${ }^{2}$ Universite Paris 6/7, CNRS IPGP, 75015 Paris, France \\ ${ }^{3}$ Solid State Physics Laboratory, ETH Zurich, 8093 Zurich, Switzerland \\ ${ }^{4}$ Institute of Electronics, Microelectronics, and Nanotechnology (IEMN), Department ISEN, CNRS-UMR 8520, B.P. 60069, \\ 59652 Villeneuve d'Ascq Cedex, France \\ ${ }^{5}$ JARA-FIT and II, Institute of Physics, RWTH Aachen University, 52074 Aachen, Germany
}

(Received 3 January 2011; revised manuscript received 25 April 2011; published 30 June 2011)

\begin{abstract}
We present Kelvin probe force microscopy measurements of single- and few-layer graphene resting on $\mathrm{SiO}_{2}$ substrates. We compare the layer thickness dependency of the measured surface potential with ab initio density functional theory calculations of the work function for substrate-doped graphene. The $a b$ initio calculations show that the work function of single- and bilayer graphene is mainly given by a variation of the Fermi energy with respect to the Dirac point energy as a function of doping, and that electrostatic interlayer screening only becomes relevant for thicker multilayer graphene. From the Raman G-line shift and the comparison of the Kelvin probe data with the ab initio calculations, we independently find an interlayer screening length in the order of four to five layers. Furthermore, we describe in-plane variations of the work function, which can be attributed to partial screening of charge impurities in the substrate, and result in a nonuniform charge density in single-layer graphene.
\end{abstract}

DOI: 10.1103/PhysRevB.83.235434

PACS number(s): 73.22.Pr, 74.20.Pq, 71.15.Mb, 63.22.Rc

\section{INTRODUCTION}

Carbon nanomaterials and in particular single-layer graphene, a truly two-dimensional (2D) solid with unique electronic $^{1,2}$ and mechanical properties, ${ }^{3}$ attract increasing interest, mainly due to possible applications in high mobility nanoelectronics, ${ }^{4-7}$ and sensor technologies. ${ }^{8}$ Since graphene only consists of surface atoms, a number of new schemes for local doping, ${ }^{9}$ surface functionalization, ${ }^{10}$ gas sensing, and atomically thin nanoelectronic circuits ${ }^{11,12}$ become accessible. In this context, the graphene-substrate interaction and, more generally, the interaction between graphene and its environment plays a crucial role as it limits the intrinsic electron mobility in graphene. ${ }^{13,14}$ The interaction with the substrate and environment is associated with the formation of disorder potential which, in turn, reduces mobilities, limits energy gaps in nanoribbons ${ }^{15,16}$ and bilayer graphene, ${ }^{17}$ and has significant impact on the noise level in graphene devices in general. ${ }^{18}$ Detailed studies elucidated substrate-induced electron-hole puddles, ${ }^{19}$ doping domains in graphene, ${ }^{20}$ and local quantum capacitances. ${ }^{21}$ Kelvin probe microscopy experiments on single- and few-layer graphene have been reported. ${ }^{22,23}$ Datta et $a$ l. $^{22}$ discussed the surface potential as a function of layer thickness and compared their result with Thomas-Fermi theory accounting for interlayer screening of the system. $\mathrm{Yu}$ et $\mathrm{al}^{23}$ have shown that the work function of singleand bilayer graphene can be widely tuned by changing the carrier density. They explain the doping-induced change in the work function using the Fermi level shift obtained from band structure calculations. Here, we report on Kelvin probe force microscopy (KFM) in ambient conditions of single-, bi-, and few-layer graphene resting on $\mathrm{SiO}_{2}$. We compare the KFM data with $a b$ initio density functional theory (DFT) calculations of the work function for substrate-doped (i.e., surface doped) graphene. The calculations take into account two effects: the substrate-induced charge on the system, and the interlayer screening, i.e., the charge redistribution among the layers. In particular, our calculations show that for singleand bilayer graphene the work function is mainly determined by the Fermi level shift with respect to the Dirac point, i.e., the substrate-induced charge, which confirms the theoretical model used by $\mathrm{Yu}$ et $a .^{23}$ The effect due to the interlayer screening becomes important for multilayer graphene thicker than bilayer. Furthermore, we compare our results with Raman G-line shifts, and discuss substrate-induced variations of the work function, which provide insight into the disorder potential at elevated doping levels in single-layer graphene.

This paper is organized as following: In Sec. II, we describe the experimental setup and present Kelvin probe measurements as well as Raman spectroscopy data. In Sec. III, we introduce the $a b$ initio DFT approach for calculating the work function as function of the number of graphene layers. Finally, we compare the experimental Kelvin probe data with the ab initio DFT calculations in Sec. IV.

\section{SETUPS AND MEASUREMENTS}

Kelvin probe measurements are performed under ambient conditions using a multimode microscope (Veeco) operated by a Nanonis SPM controller (SPECS). First, the topography of each scan line is recorded using tapping mode for distance control. In a second pass, at a constant distance from the sample (lift height $=5-50 \mathrm{~nm}$ ), we perform KFM using amplitude modulation (AM-KFM). The frequency of the applied electrical modulation, $V_{a c}$, is matched to the eigenfrequency of the cantilever, and the Kelvin controller 


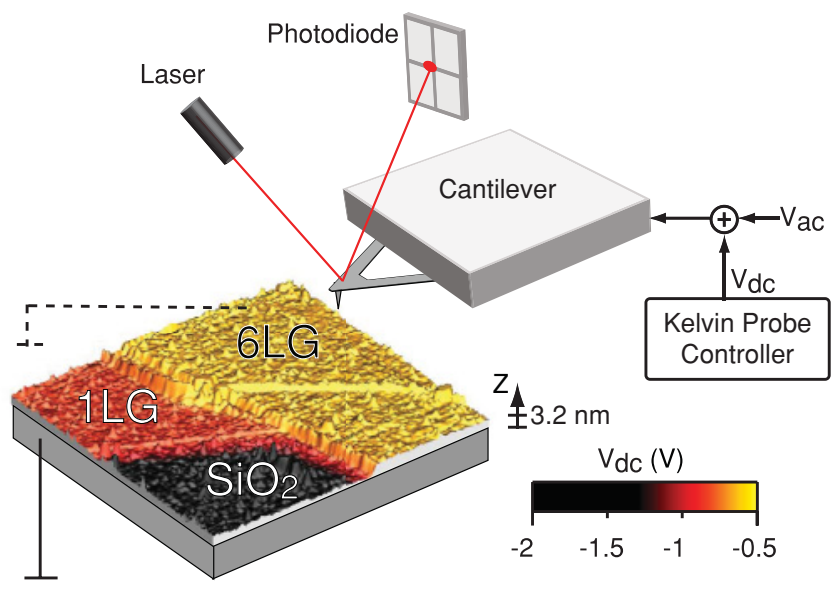

FIG. 1. (Color online) Schematic illustration of the Kelvin probe force microscopy setup used to investigate graphene flakes resting on $\mathrm{SiO}_{2}$. In the three-dimensional rendering of the topography brighter areas indicate higher recorded contact potential difference $\left(\mathrm{V}_{d c}\right)$ (see scale bar). A lower $\mathrm{V}_{d c}$ i.e. higher work function is recorded over areas of single-layer (1LG) than six-layer (6LG) regions.

cancels electrostatic forces by adjusting the tip bias voltage, $V_{d c}$, until zero amplitude is reached. ${ }^{24,25}$ Here, $V_{d c}$ compensates for potential differences between tip and sample, and if the tip work function $\Phi_{\text {tip }}$ is known, the work function of the sample is given by $\Phi_{s}=\Phi_{\text {tip }}-e V_{d c}$. The local variations of the work function, however, can be expressed independent of $\Phi_{\text {tip }}$ by $\Delta \Phi_{S}=-e \Delta V_{d c}$. An example of such a measurement is shown in Fig. 1, where the topography is mapped onto the third dimension ( $z$-axis) and the recorded surface potential $\left(V_{d c}\right)$ is color coded. Figures 2(a) and 2(c) show topography and Kelvin probe measurements of an isolated graphene flake consisting of single-layer (1LG) and bilayer ( $2 \mathrm{LG}$ ) regions [see labels in Figs. 2(a) and 2(c)]. The samples were obtained by mechanical exfoliation of bulk graphite, as described in Refs. 4 and 26. The Raman data as shown in Fig. 2(b) are acquired by using a laser excitation of $2.33 \mathrm{eV}$ through a single-mode optical fiber, whose spot size is limited by diffraction to $\sim 400 \mathrm{~nm}$. The width and shape of the 2D Raman line [see, e.g., Fig. 3(c)] confirm the number of graphene layers ${ }^{26,27}$ and single-layer regions can be well distinguished from bilayer regions in the full width at half maximum (FWHM) of the 2D line as shown in Fig. 2(b) (see areas separated by white dotted line).

In Fig. 2(d), we show a histogram analysis of the acquired Kelvin probe data over an area enclosing the graphitic flake as indicated in Fig. 2(c) (see black dashed line). Gaussian fitting reveals the average values of the measured surface potentials on the single- and bilayer graphene regions [see labels in Figs. 2(a) and 2(d)], which reproducibly show a difference of $\Delta V_{d c}^{(2-1)} \approx 66 \mathrm{mV}$ [Fig. 2(d)]. The strong scattering, i.e., the width of the distribution of the surface potential on the single- and bilayer region of $\delta V_{d c} \approx 25 \mathrm{mV}$ can most likely be attributed to an inhomogeneously charged background [see, e.g., right edge of Fig. 2(c)]. The isolated graphitic flake has been charged by bringing the metallic tip in electrical contact and applying a tip voltage $\left(V_{d c}\right)$ of either $1 \mathrm{~V}$

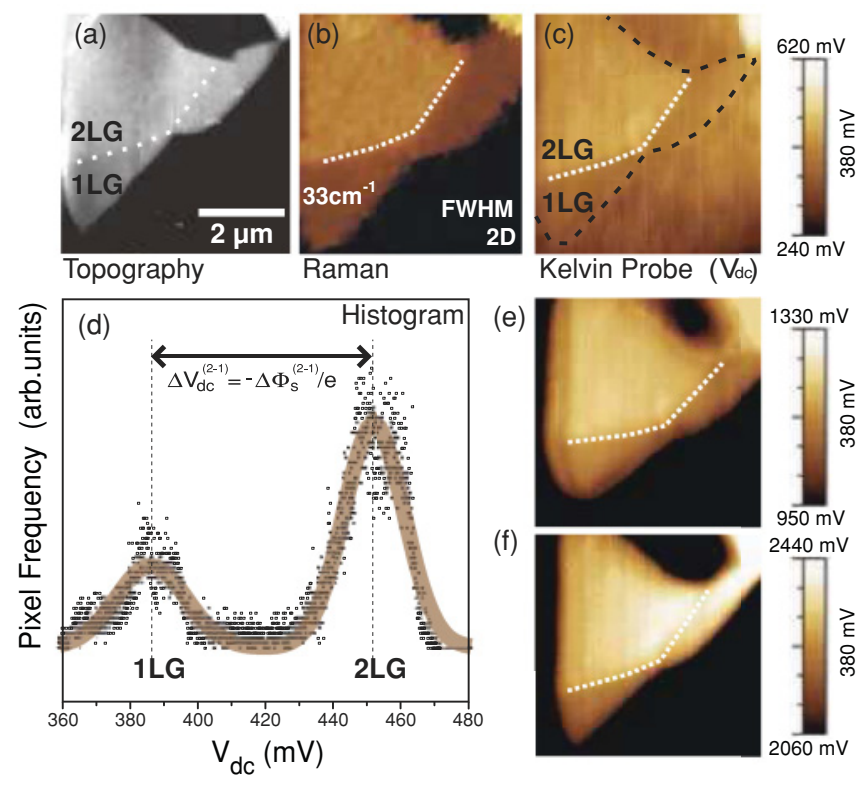

FIG. 2. (Color online) (a) AFM topography image of an isolated graphitic flake consisting of single- and bilayer regions (see labels and white dashed line along the boundaries), highlighted and proved by a confocal Raman map (b) showing the FWHM of the $2 \mathrm{D}$ Raman line. (c), (e), and (f) Kelvin probe data, i.e., surface potential measurements. (d) Histogram analysis of the acquired Kelvin probe data [taken from the area enclosed by the black dashed line shown in panel (c)]. The two peaks can be attributed to the single- (1LG) and bilayer (2LG) regions and a surface potential difference of $\Delta V_{d c}^{(2-1)} \approx$ $66 \mathrm{mV}$ is observed.

[Fig. 2(e)] or 2 V [Fig. 2(f)]. Since the highly doped Si substrate has been grounded, this corresponds to an added charge carrier density of roughly $\Delta n \approx \alpha V_{d c}$, where $\alpha=7.2 \times 10^{10} \mathrm{~cm}^{-2}$ $\mathrm{V}^{-1}$ (Ref. 4), leading to $\Delta n \approx 7.2 \times 10^{10} \mathrm{~cm}^{-2}$ and $\Delta n \approx$ $1.4 \times 10^{11} \mathrm{~cm}^{-2}$, respectively. In Figs. 2(c), 2(e), and 2(f), the relative color scale is kept constant and just the overall charge dependent offset has been adapted (see color scales). Interestingly, the surface potential difference $\Delta V_{d c}^{(2-1)}$ iswithin the error bars-not affected by the overall charging of the flake, indicating that such small charging and in particular charge rearrangements across single- and bilayer graphene regions do not strongly alter the work function difference $\Delta \Phi_{S}^{(2-1)}=-e \Delta V_{d c}^{(2-1)}$, which is in agreement with ab initio calculations as shown below.

In Fig. 3, we show measurements taken on a different sample consisting of single-, bi-, and and few-layer graphene regions with a number of layers ranging from one to six. This flake has been further electrically contacted to measure the work function independently of electrostatic influences from trapped charges within the flake. Figure 3(a) shows a confocal Raman map of the FWHM of the 2D line taken prior to electrical contact deposition. We clearly distinguish the single-layer region (left dark area) from the multilayer region (bright part). This particular flake consists of a number of regions with different layer thicknesses as shown in Fig. 3(b), where the Raman G-line intensity is plotted (arbitrary units). In Figs. 3(c) and 3(d), we show two examples of the full Raman spectra taken on two different regions [see labels 
(a)

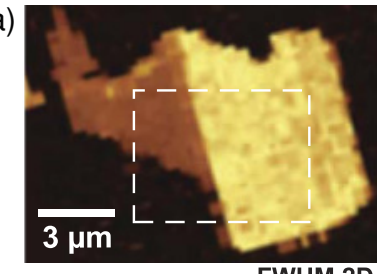

(c)

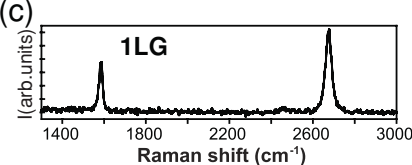

(e)

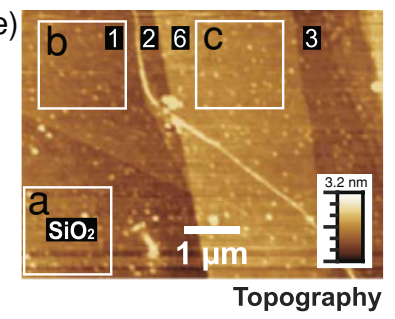

(b)

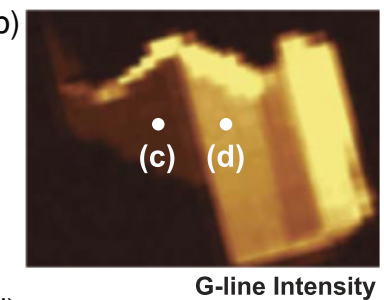

(d)

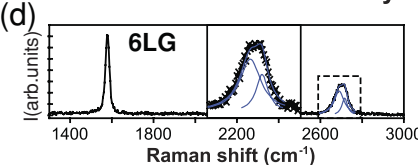

(f)

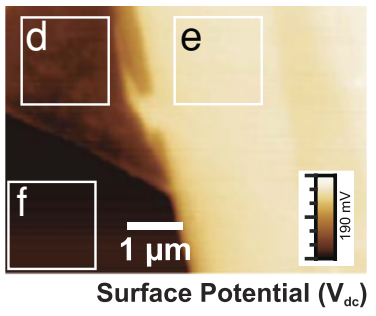

FIG. 3. (Color online) (a) 2D-FWHM Raman map of a few-layer graphene flake with sections ranging from six layers down to a single layer (taken before electrically contacting the flake). (b) Raman map of the G-line intensity where (c) and (d) are the full Raman spectra taken on two different region [see labels in (b)]. (e) The AFM topography (with inset indications of the number of layers) and (f) Kelvin probe image of the same flake after contacting [image corresponds to the dashed square in panel (a)]. The labeled boxes therein relate to Fig. 6 .

in Fig. 3(b)]. Figure 3(c), for example, shows a single-layer spectrum with a narrow 2D line (and no D line), whereas Fig. 3(d) shows a Raman spectrum of a six-layer-thick region confirmed by atomic force microscope (AFM) measurements. Topography and Kelvin probe measurements of this very same flake are shown in Figs. 3(e) and 3(f). The different layer dependent surface potential values $V_{d c}$ can be clearly identified in Fig. 3(f) [see also numbered labels in Fig. 3(e)]. We observe that the surface potential $V_{d c}$ increases with increasing layer thickness, which predicts p-doping of the sample. ${ }^{28}$ For the surface potential difference between the single- and bilayer region we find $\Delta V_{d c}^{(2-1)} \approx 68 \mathrm{meV}$, which is very close to the value reported above. However, other groups reported larger values of $\Delta V_{d c}^{(2-1)}$ (Refs. 22 and 23), but as shown in the following, this can be attributed to different doping, which strongly depends on the preparation of the sample. ${ }^{29,30}$ The three closeups of Figs. 3(e) and 3(f) (see white labeled 1.5 by $1.5 \mu \mathrm{m}$ boxes therein) will be discussed in Fig. 6 .

The observed differences in work function compared to a multilayer area $\Delta \Phi_{S}^{(N)}$ are depicted in Fig. 4 (full squares). Compared to bulk graphite we observe a difference of 150 , 82 , and $26 \mathrm{meV}$, for single-, bi-, and three-layer graphene, respectively. The particularly large scattering of $\Delta \Phi_{S}^{(2)}$ may be related to the relatively narrow bilayer region. The inset in Fig. 4 shows the measured Raman G-line shift as a function of number of layers. The doping $n$ of the sample can be determined from the G-line position $\omega_{G}$ using the calculated relations $n\left(\omega_{G}\right)$ for single-layer ${ }^{31}$ and bilayer graphene. ${ }^{32}$ The G-line shift is nearly symmetric and positive for electron

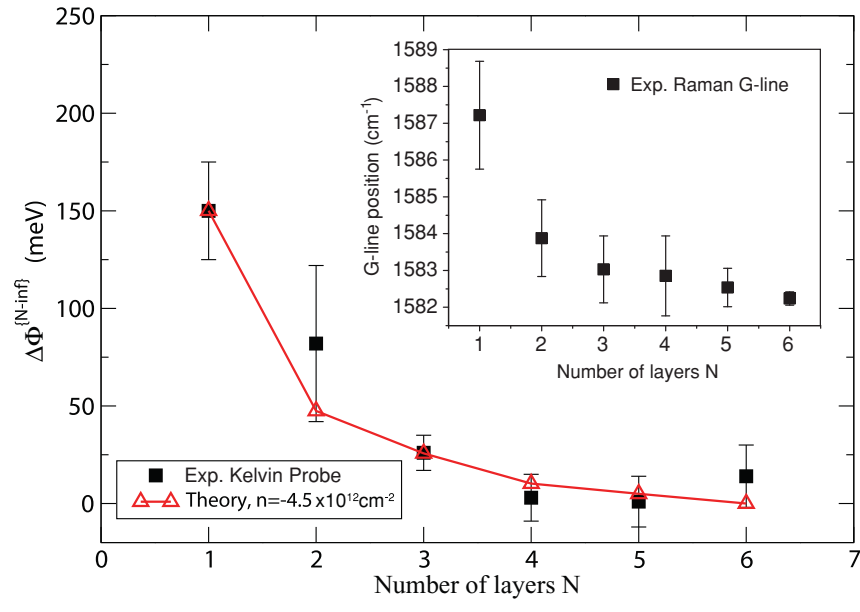

FIG. 4. (Color online) Work function differences as a function of the layer thickness (full squares), extracted by histogram analysis of the acquired Kelvin probe data. (Inset) Raman G-line shift. Triangles indicate $a b$ initio DFT calculations of the work functions for a doping level of $n=-4.5 \times 10^{12} \mathrm{~cm}^{-2}$.

and hole doping, therefore the sign of the doping cannot be extracted from the measured G-line shift. However, assuming a hole doping on this flake, we find a doping level of $n \approx$ $-(2.5 \pm 1) \times 10^{12} \mathrm{~cm}^{-2}$ for the single-layer and $n \approx-(7.5 \pm$ $1.5) \times 10^{12} \mathrm{~cm}^{-2}$ for the bilayer. Similar values can be obtained using the experimentally measured $\omega_{G}$ as a function of $n .^{20,33,34}$ These Raman measurements suggest that single- and bilayer graphene on the same sample have different doping values, which will be further discussed below. For a number of layers larger than two, the analogous relation $n\left(\omega_{G}\right)$ has not been determined yet.

\section{DENSITY FUNCTIONAL THEORY CALCULATION}

In order to compare the Kelvin probe measurements with $a b$ initio DFT calculations, we used the PWscf code of the Quantum ESPRESSO distribution. ${ }^{35,36}$ Calculations are done using the Perdew-Burke-Ernzerhof exchange correlation functiona $^{37}$ and norm-conserving pseudopotentials. Wave functions and density are described using 40 and 600 Ry planewave basis set, respectively. The electronic eigenstates are occupied with a Fermi-Dirac distribution, with an electronic temperature of $300 \mathrm{~K}$. The Brillouin zone integration is performed using a k-point grid of $(80 \times 80 \times 1)$. The experimental lattice constant $a=2.46 \AA$ of the $2 \mathrm{D}$ graphite is used and the layer-layer distance $d$ in multilayer graphene is set to $3.35 \AA$ as in graphite. The length $L$ of the supercell along $z$ is fixed in order to have a distance of about $14 \AA$ between periodic replica and a distance of about $7 \AA$ between the system and the monopole and dipole potentials region. Multilayer graphene sheets are arranged according to Bernal stacking geometry. The calculations only include surface-induced charge doping originating from the bottom or top surface of the graphitic flakes. Possible influences of surface dipoles associated to adsorbed molecules are neglected. For simplicity we assume that the dopants are on the bottom side only. The density of surface charges at the bottom side of the flake is $\sigma=-n|e|$, where $n$ is the free carrier density and $-|e|$ is the electron 
charge. Positive $n$ corresponds to electron doping and negative $n$ corresponds to hole doping. The surface charge density leads to a discontinuity of the electric field across the graphene layers equal to $\sigma / \epsilon_{0}$, where $\epsilon_{0}$ is the permittivity of the vacuum. We assume that the counter charge density is located on the bottom side of the system (e.g., in the gate electrode) such that the electric field is $\mathbf{E}=\sigma / \epsilon_{0}$ below the graphene sheet and, assuming absence of adsorbates, we set $\mathbf{E}=0$ above the flake. The electric field configuration is reproduced in our calculations by introducing monopole and dipole potentials in the edges of the super cell as described by Gava et al. ${ }^{38}$ The resulting potential energy in the direction perpendicular to the sheet is shown in Fig. 5(a) for single- and bilayer graphene (a planar average in the directions parallel to the sheets has been performed). The electric field is obtained from the derivative of the planar average, calculated in the region outside few-layer graphene where the potential energy is linear and $\mathbf{E}$ uniform. The zero electric field condition on the top side of the system is required to define the work function $\Phi_{S}$, which is the minimum energy for an electron to escape into vacuum. $\epsilon_{\infty}$ is the potential energy at infinite distance from the system [in Fig. 5(a), it is set to zero for convenience]. The work function $\Phi_{S}$ of multilayer graphene is then defined as

$$
\Phi_{S}=\epsilon_{\infty}-\epsilon_{F},
$$

where $\epsilon_{F}$ is the Fermi level.

In the insets of Fig. 5(a), we show the calculated band structure for $\mathrm{p}$-doped single- and bilayer graphene, around the $\mathrm{K}$ point in the Brillouin zone (BZ). The effect of the electric field on the system is twofold: (i) the band structure is distorted and the alignment of the Dirac point is modified with respect to the vacuum energy and (ii) the Fermi level is shifted with respect to the Dirac point because of removal or addition of charges. For the single layer, the electric field does not affect the linear crossing of the $\pi$ bands (which is dictated by the hexagonal symmetry of the system). However, for the bilayer the zero-field electric band structure displays a parabolic crossing of the $\pi$ bands at $K,{ }^{39}$ and this crossing is lifted in presence of a finite electric field.

In Fig. 5(b), we show the doping dependence of the work function $\Phi_{S}$ of single and few-layer graphene, calculated with Eq. (1), which includes both the effect of the Fermi level shift due to the induced charge and the effect due to the different screening properties of multilayer graphene. The work function for single-layer graphene shows a strong doping dependence, while its variation in $n$ decreases with increasing number of layers. This is due to the fact that the doping charge (per unit area) can distribute over different layers. Thus, the more layers there are, the lower is the Fermi level shift.

The doping dependence of $\Phi_{S}$ can be due to both, the variation of the Fermi level with respect to the Dirac point $\epsilon_{d}$, or an electric-field-induced variation of $\epsilon_{d}$ with respect to the vacuum level:

$$
\Phi_{S}=\left(\epsilon_{\infty}-\epsilon_{d}\right)+\left(\epsilon_{d}-\epsilon_{F}\right),
$$

where $\epsilon_{d}$ is calculated as the average energy of the $\pi$ bands at $\mathrm{K} .{ }^{40}$ For zero doping, our DFT calculations show that $\left(\epsilon_{\infty}-\epsilon_{d}\right)$ does not depend on the number of layers and it is (a)

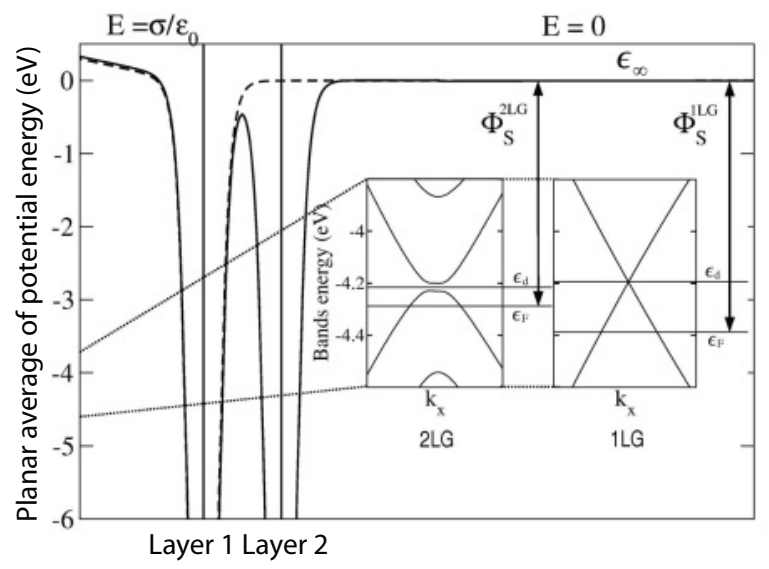

(b)
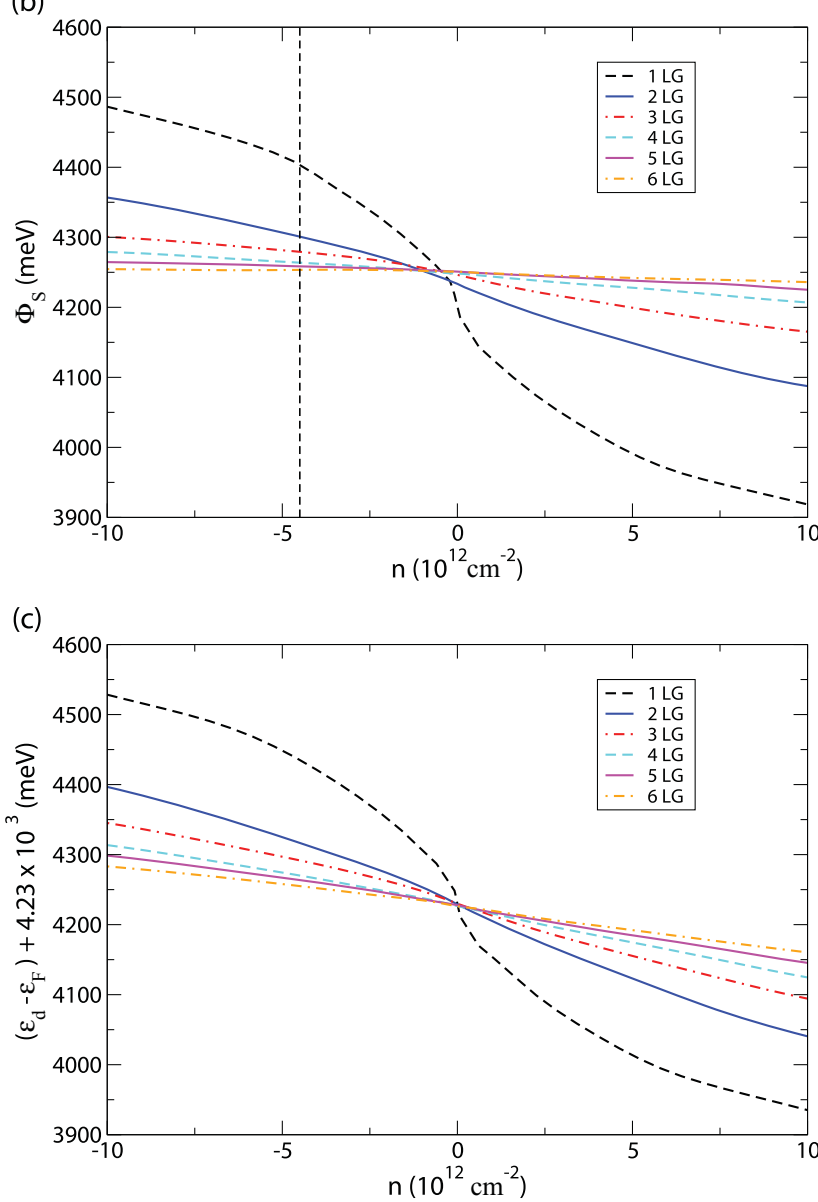

FIG. 5. (Color online) (a) Planar average of the potential energy across $1 \mathrm{LG}$ (dashed line) and 2LG (continuous line), for a doping value $n=-4.5 \times 10^{12} \mathrm{~cm}^{-2}$, calculated in DFT. (Insets) Corresponding band structures of $1 \mathrm{LG}$ and $2 \mathrm{LG}$ around the K point, illustrating the difference in work function $\Phi_{S}^{1 \mathrm{LG}}$ for single- and $\Phi_{S}^{2 \mathrm{LG}}$ for bilayer graphene. The planar average and band structures are plotted with respect to the energy in the vacuum $\epsilon_{\infty}$, here set to zero for convenience. (b) Ab initio DFT calculated work function, $\Phi_{S}$, as a function of doping concentration $n$, for multilayers graphene obtained from Eq. (1). The vertical dashed line indicates a doping value of $n=-4.5 \times 10^{12} \mathrm{~cm}^{-2}$ for which we found the best agreement with the measured work function for single-layer graphene. (c) Approximated expression of $\Phi_{S}$, as in Eq. (3). 
$\approx 4.23 \mathrm{eV}$. Assuming that $\left(\epsilon_{\infty}-\epsilon_{d}\right)$ is independent of the doping:

$$
\Phi_{S} \approx\left(\epsilon_{d}-\epsilon_{F}\right)+4.23 \mathrm{eV}
$$

The comparison between Figs. 5(b) and 5(c) [i.e., Eqs. (2) and (3)] shows that the approximated expression [Eq. (3)] well reproduces the doping dependence of the work function for single-layer graphene. For the largest values of $n$ considered in Fig. 5(c), the error in the work function difference with respect to $n=0$ is around 4\%. Equation (3) also reasonably reproduces the doping dependence of the work function for bilayer graphene, even if in this case the error is increased with respect to single-layer, being around $30 \%$. In single- and bilayer graphene, the work function is thus mainly determined by the Fermi level shift with respect to the Dirac point as a function of the charge density, justifying the analysis of $\mathrm{Yu}$ et $a l^{23}$ Our findings also explain a $\sqrt{n}$ dependence of $\Phi_{S}$ on the charge density for the single layer, and a linear dependence on $n$ for the double layer. Indeed, for the single layer

$$
\epsilon_{d}-\epsilon_{F}=-\operatorname{sign}(n) \sqrt{\pi} \hbar v_{F} \sqrt{|n|},
$$

and for the biayer

$$
\epsilon_{d}-\epsilon_{F}=-\frac{\pi \hbar^{2} n}{2 m_{1}},
$$

where $v_{F}$ is the Fermi velocity and $m_{1}$ is the effective mass. Moreover, in our DFT calculations charges are adsorbed from the bottom of the system. However, in the case of single- and bilayer graphene, we have shown that the major effect which determines the work function variation is the Fermi level shift with respect to the Dirac point, i.e., the total doping charge $n$, while the Dirac point shift with respect to the vacuum is negligible. Therefore, from this decomposition, we conclude that in the case of single- and bilayer graphene the work function results to be independent of whether such charge is adsorbed from the top or the bottom of the system. For more than two layers, the error in the work function difference with respect to zero doping $(n=0)$ becomes larger than in bilayer graphene when using Eq. (3) and, therefore, the work function cannot be interpreted as a simple shift in the Fermi energy. In particular, one can notice that for a large number of layers the work function variation with $n$ is negligible [Fig. 5(b)], because the graphene layers screen the electric field on the bottom side of the sample. This screening is neglected in Eq. (3) and therefore, for the comparison with experimental results, we have used the work function values as calculated in Eq. (1) and shown in Fig. 5(b).

\section{COMPARISON AND DISCUSSION}

\section{A. Layer dependent work function differences}

In Fig. 4, we compare the experimentally observed work function differences as a function of the layer thickness with the results of our $a b$ initio DFT calculations. The experimental results are in reasonable agreement with the $a b$ initio calculations when assuming an overall doping value of $n=-4.5 \times 10^{12} \mathrm{~cm}^{-2}$ (see triangles and continuous line). We observe that the work function difference decreases significantly for the first few-layers but it is no longer layer-dependent for systems with more than four to five layers. This is in good agreement with recent transport experiments on double-gated few-layer graphene systems, where an interlayer screening length of $1.2 \pm 0.2 \mathrm{~nm}$ has been reported. ${ }^{41}$ In contrast to the Kelvin probe data, we observe for the layer-dependent Raman G-line position (see inset in Fig. 4) a slight monotonic change even for larger layer numbers $N$. This is mainly attributed to the fact that interlayer screening does not play an important role for the Raman measurement, since all layers are contributing to the Raman signal. This is in contrast to the Kelvin probe measurement, where the surface potential is playing the crucial role and lower lying layers are partially screened. However for very few-layer graphene samples (one to three layers), where the doping-induced shift of the charge neutrality point is the dominating mechanism for shifting the work function difference (see discussion in Sec. III), the Raman data and the Kelvin probe data are in reasonably good agreement. For example, we observe an enhancement of the doping level of the bilayer section in both data sets. The origin of this elevated doping value is not known but it may be related to the relatively small bilayer graphene area on the investigated sample, where edge effects may play an important role, may also explain the rather extended error bar. However, a systematic increased doping level for bilayer graphene cannot be excluded, since it could be in agreement with recent experimental results on chemically functionalized graphene where it has been found that adsorbates bind differently to single- and bilayer graphene. $^{42}$

From Fig. 5(b), we can also notice that at the estimated p-doping values (indicated by the vertical line), the small charging $\Delta n=1.4 \times 10^{11} \mathrm{~cm}^{-2}$ induced upon contact with the biased AFM tip only leads to a minor modification of the work functions, i.e., of $\Delta V_{d c}^{(2-1)}$ as observed in our experiment and mentioned above.

\section{B. Lateral variations in the work function of single- and few-layer graphene}

From the lateral variations in the measured surface potential we can furthermore extract information about in-plane screening in few-layer graphene. In Fig. 6, we show closeup scans of the topography and surface potential recorded on $\mathrm{SiO}_{2}, 1 \mathrm{LG}$, and 6LG areas [see white boxes in Figs. 3(e) and 3(f)]. The three topography images [Figs. 6(a)-6(c)] do not show significant differences. They all exhibit the presence of contaminations resting either on the substrate [Fig. 6(a)] or on the graphene surfaces independent of the layer thickness [Figs. 6(b) and 6(c)]. In contrast, the Kelvin probe measurements [Figs. 6(d)-6(f)] show striking differences between the $\mathrm{SiO}_{2}$, the $1 \mathrm{LG}$, and the $6 \mathrm{LG}$ surface. For a better comparison, we subtract the average value from each data set and choose an identical color scale of $20 \mathrm{mV}$ and perform histogram analysis. The surface potentials measured on the 6LG are very homogeneous and scattering is mainly limited by the KFM resolution [see histogram in Fig. 6(i)]. The data taken on $\mathrm{SiO}_{2}$ [Fig. 6(d)], however, show significant local variations of the surface potential. Since no such effect was observed over the 6LG area with similar topography, we can exclude topographic artifacts in the KFM signal. The variations in the surface potential on $1 \mathrm{LG}$ [Fig. 6(e)] can be attributed to 

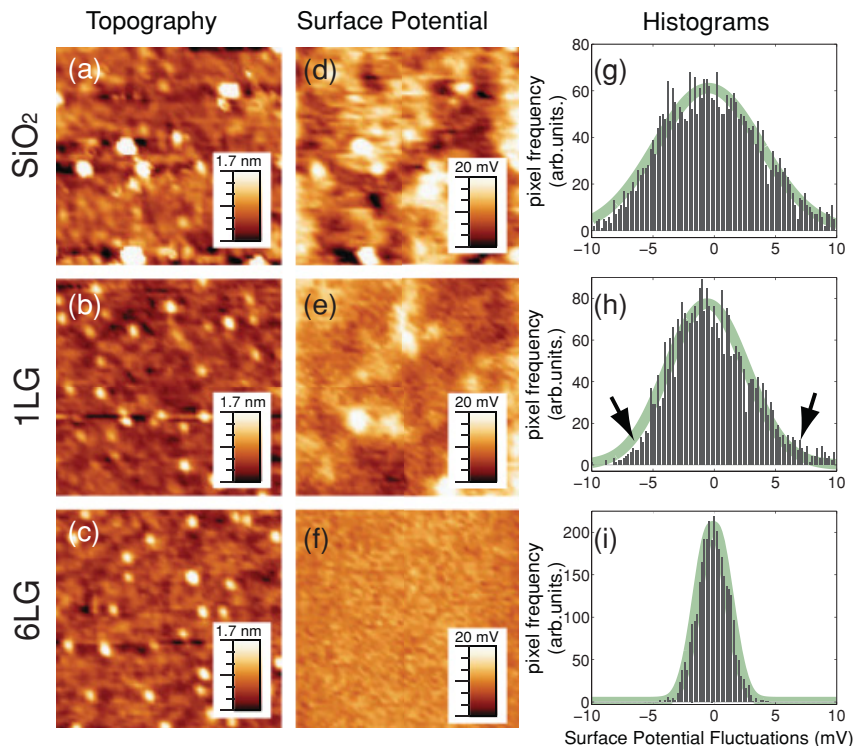

FIG. 6. (Color online) Closeup views of topography $(1.5 \times$ $1.5 \mu \mathrm{m})$ and corresponding Kelvin probe data recorded on $\mathrm{SiO}_{2}$ (a) and (d), 1LG (b) and (e), and 6LG (c) and (f) as indicated in Figs. 3(e) and 3(f). The topography images exhibit the presence of contaminations, but do not show significant differences depending on the layer thickness. The Kelvin probe measurements, in contrast, show striking differences. Whereas the work function measured on 6LG is very homogeneous, we see significant variations on $1 \mathrm{LG}$, showing that different substrate doping cannot be sufficiently screened. The width of the distribution $\delta V_{d c}^{(1)} \approx 9 \mathrm{mV}$ can be attributed to such local doping variations resulting in a charge inhomogeneity, while $\delta V_{d c}^{(6)} \approx$ $3.6 \mathrm{mV}$ lies in the range of the noise level of our KFM measurement setup. The variations are indicated by the corresponding histograms shown in panels $(\mathrm{g})$ and $(\mathrm{h})$.

partial screening of charge impurities that induce a doping. The corresponding histograms [Figs. 6(g) and 6(h)] show that single-layer graphene partially screens the impurities as expressed by a narrowing of the distribution in Fig. 6(h). For single-layer graphene, we observe an asymmetric distribution of the surface potential as highlighted by the two arrows in Fig. 6(h), whereas Figs. 6(g) and 6(i) exhibit a rather symmetric distribution. This asymmetry reflects the overall p-doping of the sample resulting in an excess of holes in the graphene layer, which allows more efficient screening of electrons. Additionally, the linear density of states suppresses the screening of more positive charge impurities in the p-doped regime [see right arrow in Fig. 6(h)]. Interestingly, we observe these strong variations in doping at elevated doping levels which consequently leads to the conclusion that significant disorder is also present at rather high carrier densities.

\section{CONCLUSION}

In summary, we presented Kelvin probe measurements on single-, bi-, and few-layer graphene flakes resting on $\mathrm{SiO}_{2}$. The Kelvin probe measurements revealed significant work function variations as a function of the number of graphene layers, in quantitative agreement with ab initio DFT computed work functions for p-doped multilayer graphene. Moreover, our calculations show that for single- and bilayer graphene the work function variation with doping is mainly due to the shift of the Fermi energy with respect to the Dirac point, while for more than two layers the effects due to interlayer screening become relevant and have to be carefully taken into account. Measured and computed work function independently lead to an interlayer screening length in the order of four to five layers. The measurements, moreover, indicate different doping values for single- and bilayer graphene on the same sample. These findings are consistent with our Raman G-line shift measurements. We observed significant variations of the surface potential on $1 \mathrm{LG}$. These variations are related to the partial screening of charge impurities in the substrate which results in a nonuniform charge density in graphene, and presently determine one of the major limitations in the performance of state-of-the-art graphene based electronic devices.

\section{ACKNOWLEDGMENTS}

The authors wish to thank D. Bishop, P. Brouwer, K. Ensslin, F. Guinea, T. Ihn, and R. W. Stark for helpful discussions and C. Hierold for providing access to the Raman spectrometer. Support by the ETH FIRST Lab and financial support by the Swiss National Science Foundation and NCCR Nanoscience are gratefully acknowledged. Calculations were performed at the IDRIS supercomputing center (Project No. 096128).
*Present address: Lawrence Berkeley National Laboratory, Molecular Foundry, 1 Cyclotron Road, Berkeley, California 94720, USA; dziegler@lbl.gov

${ }^{1}$ A. K. Geim and K. S. Novoselov, Nature Mater. 6, 183 (2007).

${ }^{2}$ A. H. C. Neto, F. Guinea, N. M. R. Peres, K. S. Novoselov, and

A. K. Geim, Rev. Mod. Phys. 81, 109 (2009).

${ }^{3}$ C. Lee, X. Wei, J. Kysar, and J. Hone, Science 321, 385 (2008).

${ }^{4}$ K. S. Novoselov, A. K. Geim, S. V. Morozov, D. Jiang, M. I. Katsnelson, I. V. Grigorieva, S. V. Dubonos, and A. A. Firsov, Science 306, 666 (2004).

${ }^{5}$ M. I. Katsnelson, Mater. Today 10, 20 (2007).

${ }^{6}$ X. Wang, Y. Ouyang, X. Li, H. Wang, J. Guo, and H. Dai, Phys. Rev. Lett. 100, 206803 (2008).
${ }^{7}$ P. Avouris, Z. Chen, and V. Perebeinos, Nat. Nanotechnol. 2, 605 (2007).

${ }^{8}$ F. Schedin, A. K. Geim, S. V. Morozov, D. Jiang, E. H. Hill, P. Blake, and K. S. Novoselov, Nature Mater. 6, 652 (2007).

${ }^{9}$ D. B. Farmer, R. Golizadeh-Mojarad, V. Perebeinos, Y.-M. Lin, G. S. Tulevski, J. C. Tsang, and P. Avouris, Nano Lett. 9, 4474 (2009).

${ }^{10}$ F. M. Koehler, N. A. Luechinger, D. Ziegler, E. K. Athanassiou, R. N. Grass, A. Rossi, C. Hierold, A. Stemmer, and W. J. Stark, Angew. Chem., Int. Ed. Engl. 48, 224 (2009).

${ }^{11}$ C. Stampfer, E. Schurtenberger, F. Molitor, J. Guettinger, T. Ihn, and K. Ensslin, Nano Lett. 8, 2378 (2008).

${ }^{12}$ J. Guettinger, C. Stampfer, S. Hellmueller, F. Molitor, T. Ihn, and K. Ensslin, Appl. Phys. Lett. 93, 212102 (2008). 
${ }^{13}$ K. I. Bolotin, K. J. Sikes, J. Hone, H. L. Stormer, and P. Kim, Phys. Rev. Lett. 101, 096802 (2008).

${ }^{14}$ X. Du, I. Skachko, A. Barker, and E. Y. Andrei, Nat. Nanotechnol. 3, 491 (2008).

${ }^{15}$ K. Todd, H.-T. Chou, S. Amasha, and D. Goldhaber-Gordon, Nano Lett. 9, 416 (2009).

${ }^{16}$ C. Stampfer, J. Guttinger, S. Hellmuller, F. Molitor, K. Ensslin, and T. Ihn, Phys. Rev. Lett. 102, 056403 (2009).

${ }^{17}$ J. B. Oostinga, H. B. Heersche, X. Liu, A. F. Morpurgo, and L. M. K. Vandersypen, Nature Mater. 7, 151 (2008).

${ }^{18}$ Y.-M. Lin and P. Avouris, Nano Lett. 8, 2119 (2009).

${ }^{19}$ J. Martin, N. Akerman, G. Ulbricht, T. Lohmann, J. Smet, K. von Klitzing, and A. Yacoby, Nat. Phys. 4, 144 (2008).

${ }^{20}$ C. Stampfer, F. Molitor, D. Graf, K. Ensslin, A. Jungen, C. Hierold, and L. Wirtz, Appl. Phys. Lett. 91, 241907 (2007).

${ }^{21}$ F. Giannazzo, S. Sonde, V. Raineri, and E. Rimini, Nano Lett. 9, 23 (2009).

${ }^{22}$ S. S. Datta, D. R. Strachan, E. J. Mele, and A. T. C. Johnson, Nano Lett. 9, 7 (2008).

${ }^{23}$ Y.-J. Yu, Y. Zhao, S. Ryu, L. E. Brus, K. Kim, and P. Kim, Nano Lett. 9, 3430 (2009).

${ }^{24}$ M. Nonnenmacher, M. P. O'Boyle, and H. K. Wickramasinghe, Appl. Phys. Lett. 58, 2921 (1991).

${ }^{25}$ H. O. Jacobs, H. F. Knapp, S. Mueller, and A. Stemmer, Ultramicroscopy 69, 39 (1997).

${ }^{26}$ D. Graf, F. Molitor, K. Ensslin, C. Stampfer, A. Jungen, C. Hierold, and L. Wirtz, Nano Lett. 7, 238 (2007).

${ }^{27}$ A. C. Ferrari, J. C. Meyer, V. Scardaci, C. Casiraghi, M. Lazzeri, F. Mauri, S. Piscanec, D. Jiang, K. S. Novoselov, S. Roth, and A. K. Geim, Phys. Rev. Lett. 97, 187401 (2006).

${ }^{28}$ T. Filleter, K. V. Emtsev, T. Seyller, and R. Bennewitz, Appl. Phys. Lett. 93, 133117 (2008).

${ }^{29}$ Y. J. Kang, J. Kang, and K. J. Chang, Phys. Rev. B: Condens. Matter 78, 115404 (2008).
${ }^{30}$ J. Sabio, C. Seoánez, S. Fratini, F. Guinea, A. H. Castro Neto, and F. Sols, Phys. Rev. B: Condens. Matter 77, 195409 (2008).

${ }^{31}$ M. Lazzeri and F. Mauri, Phys. Rev. Lett. 97, 266407 (2006).

${ }^{32}$ P. Gava, M. Lazzeri, A. M. Saitta, and F. Mauri, Phys. Rev. B: Condens. Matter 80, 155422 (2009).

${ }^{33}$ J. Yan, Y. Zhang, P. Kim, and A. Pinczuk, Phys. Rev. Lett. 98, 166802 (2007)

${ }^{34}$ J. Yan, E. A. Henriksen, P. Kim, and A. Pinczuk, Phys. Rev. Lett. 101, 136804 (2008).

${ }^{35}$ P. Giannozzi, S. Baroni, N. Bonini, M. Calandra, R. Car, C. Cavazzoni, D. Ceresoli, G. L. Chiarotti, M. Cococcioni, and I. Dabo et al., J. Phys.: Condens. Matter 21, 395502 (2009).

${ }^{36}$ S. Baroni, A. Dal Corso, S. de Gironcoli, P. Giannozzi, C. Cavazzoni, G. Ballabio, S. Scandolo, G. Chiarotti, P. Focher, A. Pasquarello, K. Laasonen, A. Trave, R. Car, N. Marzari, and A. Kokalj, "Quantum espresso: open-source package for research in electronic structure, simulation, and optimization," 2005 (see, http://www.quantum-espresso.org/).

${ }^{37}$ J. P. Perdew, K. Burke, and M. Ernzerhof, Phys. Rev. Lett. 77, 3865 (1996).

${ }^{38}$ P. Gava, M. Lazzeri, A. M. Saitta, and F. Mauri, Phys. Rev. B: Condens. Matter 79, 165431 (2006).

${ }^{39}$ S. Latil and L. Henrard, Phys. Rev. Lett. 97, 036803 (2008).

${ }^{40}$ Note that the application of the electric field induces, for instance in bilayer graphene, the opening of a gap in the band structure and therefore a variation of the density of states, which influences the work function. This effect is included in our DFT calculations, where both terms in Eq. (2) are computed taking into account the modification of the band structure and of the density of states induced by the electric field.

${ }^{41}$ H. Miyazaki, S. Li, A. Kanda, and K. Tsukagoshi, Semicond. Sci. Technol. 25, 034008 (2010).

${ }^{42}$ F. Koehler, A. Jacobsen, K. Ensslin, C. Stampfer, and W. Stark, Small 6, 1125 (2010). 